CHICAGO-Although scientists have been able to transfer foreign genes into plant cells for several years, only a few foreign genes have been expressed successfully so far. Researchers at Monsanto (St. Louis, MO) have succeeded in adding another gene from an unlikely source.

Robert Horsch, research group leader in Monsanto's plant molecular biology division, reported here at the American Chemical Society's 190th meeting that his group has successfully expressed a mouse gene in petunias. The mouse gene, dihydrofolate reductase, confers methotrexate resistance: transformed plant cells grow in medium containing levels of methotrexate that kill control cells. Biochemical assays confirmed that the mouse gene had been incorporated into the petunia genome.

To obtain expression of the mouse gene, Horsch had to splice it to plant promoters to initiate transcription. He chose a constitutive cauliflower mosaic virus promoter, which functions normally in plant cells.

Investigators originally had difficulty inserting foreign genes into plant cells because of the size and complexity of the Ti plasmid, which is harbored by Agrobacterium tumefaciens. The creation of intermediate vectors solved this problem. Intermediate vectors are small plasmids containing fragments derived from T-DNAthe piece of the $\mathrm{Ti}$ plasmid that is transferred to the plant during infection. The Ti plasmid is tumorigenic: the T-DNA codes for opines, a class of tumor-specific compounds. However, these sequences can be deleted: as long as the borders of the T-DNA remain intact, transfer will still occur.

The Split End Vector (SEV) system developed by Horsch, Robert Fraley, and co-workers, is described in detail in Bio/Technology, 3: 629, July '85. pMON200, currently Monsanto's favorite split end vector, contains a synthetic DNA multilinker with multiple unique restriction sites for the convenient insertion of foreign DNA sequences; it also contains a selectable marker (neomycin phosphotransferase) that confers resistance to kanamycin and a scorable marker (nopaline synthase) that produces an easily assayed metabolite, nopaline. The vector contains a 25 base pair direct repeat which forms the boundaries of the DNA to be transferred.

These intermediate vectors can shuttle from Escherichia coli to Agrobacterium tumefaciens. They are con-

\author{
IMAGE \\ UNAVAILABLE FOR \\ COPYRIGHT \\ REASONS
}

Monsanto's newest high-tech tool.

structed in $E$. coli, where genetic manipulations are straightforward, and then transferred to $A$. tumefaciens by traditional mating. In vivo recombination replaces the T-DNA fragment of the resident $\mathrm{Ti}$ plasmid with the mutated version containing the foreign gene. Co-integrate formation results in a selectable, avirulent T-DNA. The T-DNA is then transferred to the plant by co-cultivation of $A$. tumefaciens with surface-sterilized leaf disks cut with a hole punch, thus creating a port of entry at the edges. Because only T-DNA is transferred to the plant, the transformants are stable.
The leaf disks are then transferred to shoot regeneration medium containing kanamycin: shoot regeneration occurs in two to four weeks.

The SEV system has also been used to achieve tissue-specific expression of foreign genes. Horsch says that Roger Beachy's group at Washington University (St. Louis, MO) has used the SEV system to transfer the gene for the $7 \mathrm{~S}$ subunit of a soybean storage protein into petunias. The gene is expressed only in the seed-where it should be-and not in other organs, such as the leaves.

-Jennifer Van Brunt

\title{
BIO/TECHNOLOGY STOCK INDEX
}

NEW YORK-The Bio/Technology Index of Specialty firms stood at 966 as of the close of trading on October 8. This was down slightly from its level of 987 on July 9, 1985. The Index, which represents a weighted average of stock prices of 23 publicly traded biotech companies, was set originally at 1000 in July of 1983.

In the three months since the last publication of the Index (Bio/Technology 3:684, August 1985), most stock prices remained fairly constant. Genentech stock price is down slightly (to around \$43), with the disclosure of possible side effects of tumor necrosis factor. Biogen is up to over $\$ 10$ after it hired a new chief executive officer,
James L. Vincent, from Allied-Signal. The proposed acquisition of Hybritech by Eli Lilly was enough to boost Hybritech's stock to almost $\$ 28$ as of October 8. Although this acquisition-in a pervading climate of acquisitions in many industries-has raised the question of other possible biotech purchases, most analysts do not foresee such an occurrence. The deciding factor could be that many specialty firms are already tied to one or more larger companies through equity and R\&D arrangements. If anything, then, future acquisitors could be the companies that have already made sizable investments in the specialty firms. $\quad$-Arthur Klausner 